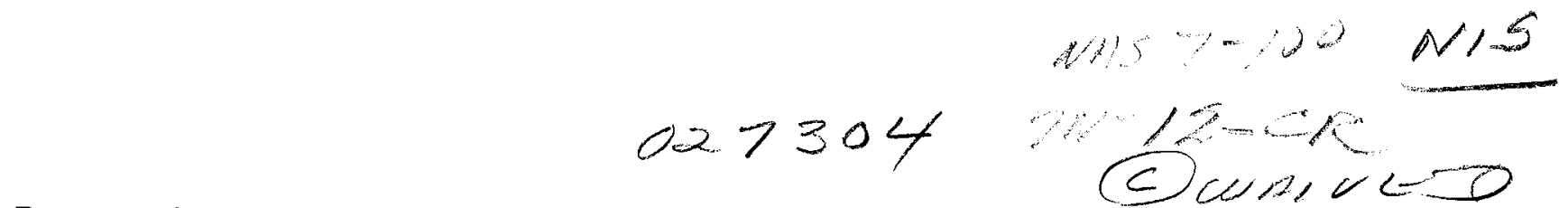

\section{Demonstration of a High-Fidelity Predictive/Preview Display Technique for Telerobotic Servicing in Space}

\author{
Won S. Kim and Antal K. Bejczy
}

\begin{abstract}
A highly effective predictive/preview display technique for telerobotic servicing in space under several seconds communication time delay has been demonstrated on a large laboratory scale in May 1993, involving the Jet Propulsion Laboratory as the simulated ground control station and, 2500 miles away, the Goddard Space Flight Center as the simulated satellite servicing set-up. The technique is based on a highfidelity calibration procedure that enables a high-fidelity overlay of 3-D graphics robot arm and object models over given 2-D TV camera images of robot arm and objects. To generate robot arm motions, the operator can confidently interact in real time with the graphics models of the robot arm and objects overlaid on an actual camera view of the remote work site. The technique also enables the operator to generate high-fidelity synthetic TV camera views showing motion events that are hidden in a given TV camera view or for which no TV camera views are available. The positioning accuracy achieved by this technique for a zoomed-in camera setting was about $\pm 5 \mathrm{~mm}$, well within the allowable $\pm 12 \mathrm{~mm}$ error margin at the insertion of a $45 \mathrm{~cm}$ long tool in the servicing task.
\end{abstract}

\section{INTRODUCTION}

There is a genuinely growing interest to reduce extravehicular activity (EVA) astronauts time for servicing operations in low Earth orbit. One way to accomplish this is to introduce ground-controlled servicing by using telerobotic devices and techniques. In such operations, however, there is an unavoidable two-way communication time delay between a ground control station and the telerobotic device working in low Earth orbit. When the existing NASA telecommunication facilities are utilized, this round-trip communication time delay can be expected to vary between four to eight seconds. This size of time delay prevents any effective closed-loop control interaction between the ground operator and the telerobotic device.

Due to the very nature of satellite or other space servicing in low Earth orbit, "blind" (that is, fully pre-programmed and visually not supervised) automation is not possible for such tasks, and fully autonomous performance of such tasks is still far down the road. There is a need, therefore, to consider and apply an operatorinteractive remote control method enabling an efficient crossing of the communication time delay barrier and still preserving operation reliability and safety.

Typical teleoperation tasks require the operator's visual and kinesthetic presence (real-time visual and force-feeling attention) to guide and control the remote robot arm's actions. Control methods and schemes have been proposed and analyzed both theoretically and experimentally in the past twenty or so years to compensate in real time for the operator's visual and kinesthetic absence in timedelayed teleoperation. (The different methods are reviewed elsewhere in this special issue; cf. [5]). There are two important and promising

Manuscript received April 12, 1993. This work was performed at the Jet Propulsion Laboratory, California Institute of Technology, under contract with the National Aeronautics and Space Administration. Several people contributed to the success of the demonstration experiments.

The authors are with the Jet Propulsion Laboratory, California Institute of Technology, Pasadena, CA 91109.

IEEE Log Number 9212403. schemes for enhancing telemanipulation capabilities under communication time delay conditions: predictive/preview displays and shared compliance control. For details, see above quoted reference and also [1], [2], and [3].

In this paper, we briefly present the demonstration and evaluation of a space telerobotic servicing task performed under communication time delay on a large laboratory scale in May, 1993. In the demonstration, a high-fidelity predictive/preview display technique developed at the Jet Propulsion Laboratory (JPL) was used combined with wrist force sensor referenced compliance control implemented at the other NASA center (Goddard Space Flight Center, GSFC) participating in the demonstration experiments. In the demonstration, JPL acted as the operator site simulating the ground control station, and GSFC, more than 2500 miles away from JPL, acted as the remote work site with a life-size satellite servicing task mock-up. During the demonstration experiments, TV camera images from the GSFC work site were sent back to the JPL control station over the NASA Select Satellite TV channel at live video rate ( 30 frames/s). Command and control data from the JPL control station to the GSFC work site and execution status and sensor data from GSFC to JPL were sent as "data blocks" through the Internet computer communication network.

First, we briefly describe the task and the task set-up at GSFC, followed by a brief summary of the salient features of the high-fidelity predictive/preview display technique developed at JPL and used in the joint JPL-GSFC demonstration experiments. Then, we briefly summarize the results. The conclusion will also indicate planned future work.

\section{Remote Servicing Task Set-Up}

The servicing demonstration task to be performed at GSFC, remotely controlled from JPL, was the exchange of a nearly four feet tall, four feet wide and two feet deep exchangeable module (called Orbital Replacement Unit, ORU) on a satellite mock-up. This module exchange task was originally designed to be performed by astronauts working in pressurized space suits in the Space Shuttle cargo bay. A robot arm (Robotics Research Corporation K-1607) was placed in front of the exchange module of the satellite's life-size set-up. The arm was equipped with an eighteen inch (about $45 \mathrm{~cm}$ ) long power screwdriver (called lightweight servicing tool, LST), which also could be rigidly connected to the exchange module to remove the module from the satellite. The basic robot control task was to insert the power screwdriver through an eighteen inch long hole to reach the module's latching mechanism at the module's back plane, then to unlatch the module from the satellite's frame, and rigidly connect the module to the robot arm in order to remove the module from the satellite. The placement of a new module back to the satellite's frame followed the reverse sequence of actions. The RRC K-1607 arm of GSFC was equipped with a 6-dof wrist force sensor. The LST and the "new" ORU module were equipped with GSFC's capaciflector proximity sensor system.

The remote control from JPL utilized three fixed TV camera settings at the GSFC test site: the first one was located at the base of the robot arm looking straight ahead towards the exchange module in the satellite. The second one was located at the right and perpendicular to the first camera view at nearly the same elevation. The third one was placed halfway in between and about 35 degrees 
above the previous two camera settings to provide an oblique view of the work scene. Two zoom settings were used for the third camera.

The ORU changeout task scenario used in the remote servicing demonstration had the following sequence:

1) Move the arm from the starting position to a position where the LST tip is about $20 \mathrm{~cm}$ in front of the entrance of the hole on the ORU module.

2) Move the LST to the immediate entrance of the hole.

3) Insert the LST.

4) Latch the LST to the ORU.

5) Turn on the power tool to loosen the screw.

6) Pull out the ORU by $5 \mathrm{~cm}$.

7) Continue to withdraw the ORU so that it is about $15 \mathrm{~cm}$ apart from the satellite.

8) Move the ORU to a stow position.

9) Move the ORU back to $15 \mathrm{~cm}$ in front of the satellite frame.

10) Align the ORU for insertion.

11) Insert the ORU.

12) Turn on power tool to tighten the screw.

13) Unlatch the LST from the ORU.

14) Pull out the LST to about $20 \mathrm{~cm}$ away from the ORU.

15) Finally, move the arm back to the starting position.

To compensate in real time for the operator's time-delayed visual perception of the task, a high-fidelity predictive/preview display technique was used. This technique provides a high-fidelity overlay of computer graphics represented virtual reality over TV camera views represented actual reality. The operator generated the overlaid robot graphics image motions by a hand controller or by computer control algorithms. Then the operator visually verified the correctness of the generated robot motions through previewing the simulated robot graphics image motions embedded into the monitor of an actual TV camera image of the work scene. Once verified, the recorded motion command is sent over to the GSFC robot control system for execution there. The robot motion execution during the actual contact phase of tool or module insertion or removal actions is aided by automatic compliance control referenced to wrist force sensor data, implemented at GSFC. This compensates in real-time for the operator's kinesthetic absence. Capaciflector proximity sensor data can play the role of automatically preventing unforeseen or accidental contact errors during near-contact but still in free-space robot motion.

\section{High-FidELITY MATCHING OF VIRTUAL REALITY TO THE ACTUAL ONE}

The process of matching the virtual reality, represented by computer graphics images, to the actual reality, as shown on TV camera images, is composed of four major high-fidelity technical components in the time-delayed telemanipulation method developed at JPL. First, the method requires the construction of high-fidelity 3-D graphics models of both the robot arm and the objects of interest for robot manipulator tasks. Second, which is the cornerstone of the method, the 3-D graphics models are calibrated with high-fidelity to the given 2-D TV camera views that cover the sight of both the robot arm and the objects of interest. Third, which is a basic requirement of the method, the calibrated graphics models are overlaid with high fidelity over the actual robot arm and object images as shown to the operator on the TV camera monitor screen. Fourth, the method requires control of the motion of the robot arm graphics image on the screen by the same control software that controls the motion of the real robot, in order to assure high fidelity in the formulation of motion commands.

This time-delayed telemanipulation method was designed to provide a high visual confidence to the operator when the operator interacts with graphics images in real time in generating, that is, in predicting, or in previewing manually or computer generated robot arm motion before the actual robot arm motion takes place. This method goes beyond the general practice which uses graphics models displayed separately from actual TV views. Our calibrated overlay method in essence provides visual verification and updates for the validity of graphics models for an actual live scene. The calibrated virtual reality display system can also provide synthetic TV camera views to the operator. Synthetic TV camera views can make critical motion events or robot arm position and alignment states visible which otherwise would be hidden from the operator in a given TV camera view or for which no real TV camera views are available. The calibrated virtual reality method also enables the placement of calibrated 3-D target symbols over the live TV video helping the real-time visual guidance/control and real-time visual verification by the operator.

Construction of high-fidelity graphics models is possible through state-of-the-art commercial graphics software systems. However, no commercial graphics software system is available which is easily compatible with the calibration and overlay requirements in our timedelayed telemanipulation method. Therefore, a graphics modeling software system (called HYDRA) was developed in-house in the past few years to have an integrated modeling, calibration, and overlay working system.

\section{A. Camera Calibration}

Accurate matching of graphics models to the actual task environment based on TV. camera views requires camera calibration and object localization. Although there are several camera calibration and object localization techniques, none of them specifically address the practical problem of calibration of graphics models for overlay on live video for predictive/preview display applications. Therefore, a reliable operator-interactive camera calibration procedure was developed [4]. In this procedure, the robot arm itself is used as a calibration fixture. The operator enters the correspondence information between 3-D graphics model points and 2-D camera image points of the arm to the computer by repeatedly clicking with a mouse a graphics model point and then its corresponding image point for each corresponding pair. (That is, the current calibration is based on a point-to-point mapping technique; the selected points are visually sharply defined characteristic points.) To improve the calibration accuracy over the normal operating space of the arm, the operator enters the corresponding data points for several different poses of the arm within the same camera view. Thereafter, the system computes the camera calibration parameters. This calibration procedure is repeated for each of the camera views that are needed for a given task.

The computation of the camera calibration parameters is based on the ideal pinhole model of image formation by the camera. Under this assumption, the image format of the camera is described by a linear perspective projection, and the relation between 3-D graphics model points and the corresponding 2-D TV camera image points can be described by a single linear $4 \times 3$ calibration matrix. In general, it would be physically more accurate to consider the second-order nonlinear radial distortion of the camera lens optics in the model of camera image formation for calibration purposes. However, this more elaborate model leads to a nonlinear projection which, in general, is not supported by real-time 3-D graphics workstations. For practical reasons, therefore, the ideal pinhole model was used in the camera calibration procedure for overlaid preview/predictive displays.

In our implementation, a Silicon Graphics workstation (IRIS 4D/310 VGX) with a square-pixel resolution monitor and with a VideoLab Board are used for the video image capture and for the calibrated graphics overlay on the live video image. In computing camera parameters using the above hardware, we further assumed 
that i) the camera optical axis is perpendicular to the image plane, passing through the center of the camera view, and ii) the viewpoint of the graphics overlay window coincides with the full size of the camera view window. We note here that the final camera calibration matrix defines the position, orientation, and field of view angle of the camera.

The actual camera calibration computations in our method can be carried out by a combination of linear and nonlinear least-squares algorithms. The linear algorithm in general does not guarantee the orthonormality of the rotation matrix, providing only an approximate solution. The nonlinear algorithm provides the least-squares solution that satisfies the orthonormality of the rotation matrix, but requires a good initial guess to converge to the desired least-squares solution without a very time-consuming random search. When an approximate solution is known, one can start with the nonlinear iterative algorithm directly by using the approximate solution as an initial guess. When an approximate solution is unknown, however, the linear algorithm is used to find first an approximate solution, and then the nonlinear algorithm is applied.

\section{B. Object Localization}

In our method of high-fidelity matching of virtual reality to the actual one, the operator performs object localization after the calibration of all desired camera views. For determining the object 3-D pose from a given 2-D camera view, several algorithms are available in the literature. In our procedure, a projection-based linear/nonlinear algorithm is extended to allow object localization for any number of multiple camera views.

The operator's interactive data entry procedure to feed inputs to the extended object localization algorithms is essentially identical to the one for camera calibration, except that the operator enters corresponding data points for an object (not the robot arm this time) with several different camera views. Again, a combination of linear and nonlinear algorithms is used for computing the object pose (position and orientation), and the computational procedure depends upon whether an approximate initial solution is known. More details on the camera calibration and object localization applied in our high-fidelity predictive/preview display technique can be found in [4].

Once the camera calibration and object localization are completed, the graphics models of both the robot arm and the object can be overlaid with high fidelity on the corresponding actual video images in a given video camera view. The arm and object graphics models can be overlaid in a wire-frame or in a solid-shaded polygonal rendering, with varying levels of transparency, providing different visual effects to the operator for different task details. The hidden lines can be removed or retained by the operator, dependent on the information needs in a given task. In many cases, retained hidden lines can provide very useful and needed information to the operator.

\section{Motion Control of Robot Arm's Graphics Image}

The idea with the high-fidelity graphics overlay image is that the operator can interact with it visually in real time when manually generating motion commands or when previewing computer generated trajectories, and doing it against the live video scene on a monitor within one perceptive frame. Thus, this method would compensate in real-time for the operator's time-delayed visual perception. Typically, the geometric dimensions of a monitor and geometric dimensions of the real work scene shown on the monitor are quite different. For instance, an 8-inch long trajectory on a monitor can correspond to a 24-inch long trajectory in the actual work space, that is, three times longer than the apparent trajectory on the monitor screen. Therefore, in order to preserve fidelity between previewed graphics arm image

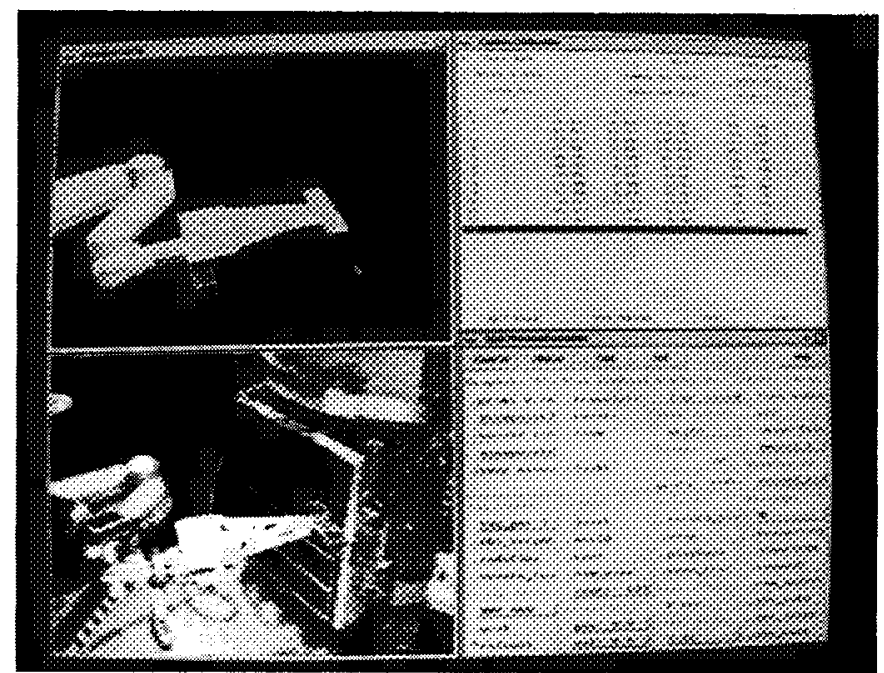

Fig. 1. Graphics user interface during robot arm graphics image calibration.

and actual arm motions, all previewed actions on the monitor were scaled down very closely to the expected real motion rate of the arm hardware. The manually generated trajectories were also previewed before sending the motion commands to the GSFC control system in order to verify that all motion data were properly recorded. Preview displays contribute to operational safety. In order to eliminate the problem associated with the varying time delay in data transfer, the robot motion trajectory command is not executed at the GSFC control system until all the data blocks for the trajectory are received.

An element of fidelity between graphics arm image and actual arm motion was given by the requirement that the motion of the graphics image of the arm on the monitor screen be controlled by the same software that controls the motion of the actual arm hardware. This required the implementation of the GSFC control software in the JPL graphics computer.

A few seconds after the motion commands were transmitted to GSFC from JPL, the JPL operator could view the motion of the real arm on the same screen where the graphics arm image motion was previewed. If everything went well, the image of the real arm followed the same trajectory on the screen that the previewed graphics arm image motion previously described, and the real arm image motion on the screen stopped at the same position where the graphics arm image motion stopped earlier. After completion of robot arm motion, the graphics images on the screen were updated with the actual final robot joint angle values. This update eliminates accumulation of motion execution errors from the graphics image of robot arm, and retains graphics robot arm position fidelity on the screen even after the completion of a force sensor referenced compliance control action.

The demonstration task involved was to connect a large payload to the robot arm, move it away from the satellite frame and then place a new payload (the same payload in our demo) back to the original position of the previous payload. This required connecting/disconnecting robot arm and payload graphics images, and to graphically simulate motion of payload held by the robot arm. If the robot arm deflection caused by the weight of a payload is not modeled, that can decrease the graphics fidelity of the payload graphics model trajectory and the graphics fidelity of the final position of the payload graphics model on the screen. This can cause problems when the payload has to be inserted under tight geometric tolerances. We encountered this problem during the demonstration experiments and compensated for it by a trial and error approach. 


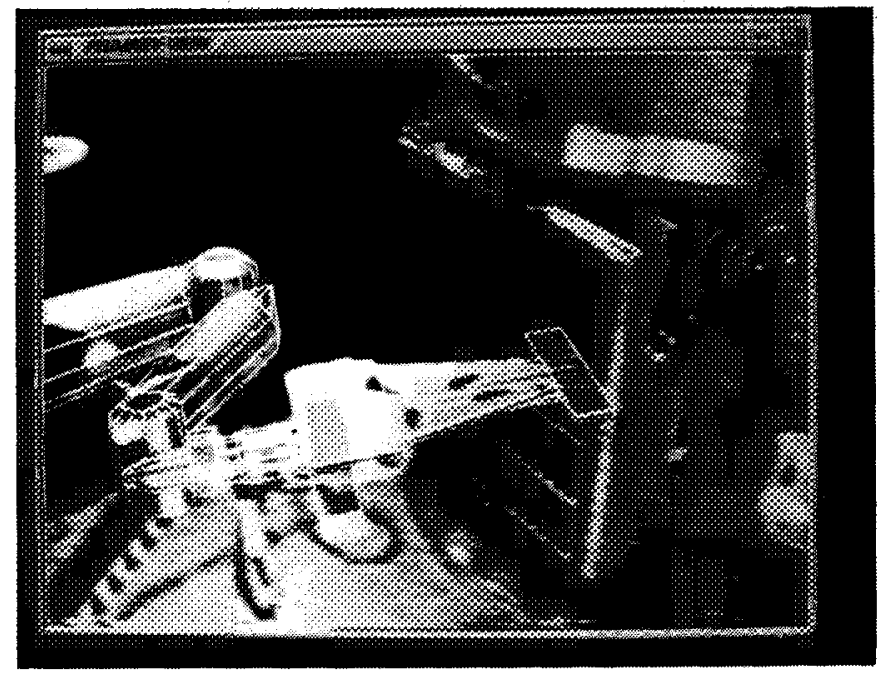

Fig. 2. A calibrated robot arm overlay in wire-frame rendering.

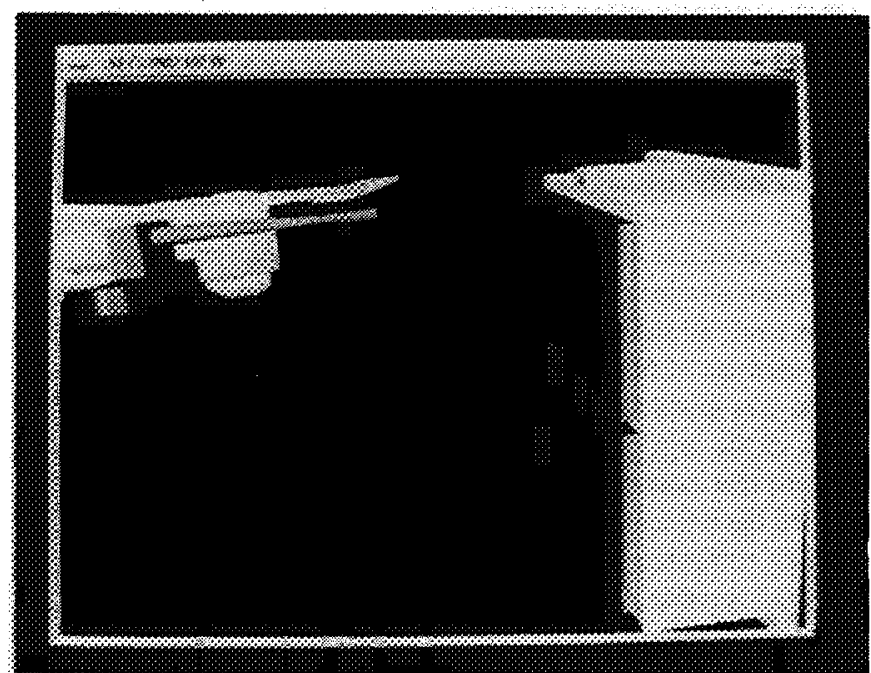

Fig. 3. A synthetic TV view of the configuration shown in Fig. 2, after object localization.

\section{Operator Interface}

There is a wealth of computer related operator activities in a system that 1 ) intends to match virtual reality to the actual one with high fidelity and 2) is expected to remotely control a robot together with its live video/graphics system and with its selectable control modes. Therefore, a useful operational operator interface requires careful design considerations. We developed a graphical operator interface system using two Silicon Graphics workstations and one NTSC video monitor. The primary workstation (IRIS $4 \mathrm{D} / 310 \mathrm{VGX}$ ) is used for preview/predictive displays and for various graphical user interfaces (GUI's) in a four-quadrant format. The second workstation (IRIS$4 \mathrm{D} / 70 \mathrm{GT}$ ) is solely used for sensor data display. More on the operator interface can be found in [4].

Fig. 1 shows the four-quadrant GUI during the arm calibration process. Fig. 2 shows the corresponding calibrated overlay result. Fig. 3 shows a synthetic TV view of the same configuration that is shown in Fig. 2, after object localization. Note that this synthetic TV view tells much more than Fig. 2 about the relative distance between tool tip and hole. Fig. 4 shows a display situation for previewing the end point of a generated motion for tool insertion. Fig. 5 shows the

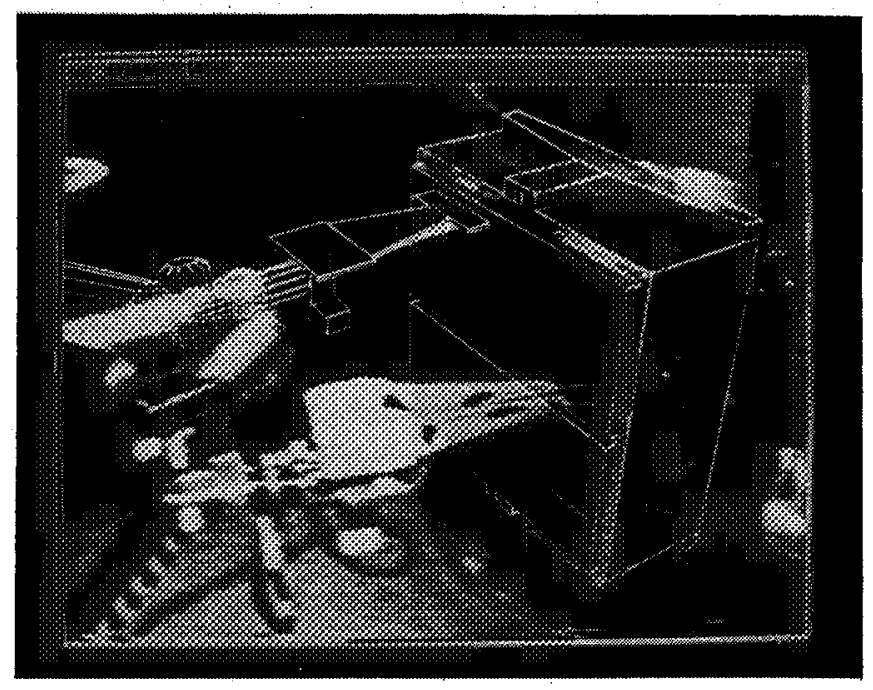

Fig. 4. Predictive/preview display of end point of first motion task including object overlay in wire-frame rendering.

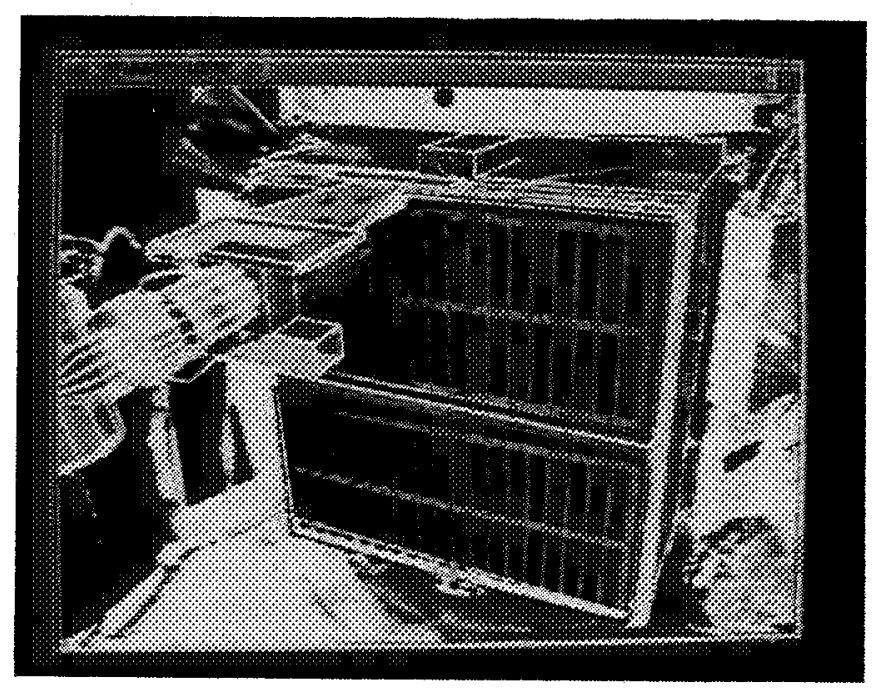

Fig. 5. Same configuration as in Fig. 4, but after motion execution and from a different $\mathrm{TV}$ camera view.

same preinsertion status from a different camera view, while Fig. 6 shows it in a zoomed-in mode.

\section{RESULTS}

Four camera views were calibrated for the May 1993 JPL-Goddard remote servicing demonstration. In each camera calibration, the operator typically entered about 15 to 30 data points in total from 3 or 4 different arm poses. For both the side-view and oblique-view cameras, the $y$-axis field-of-view angles were approximately fovy $=$ $32^{\circ}$, and the average calibration errors between the projections of 3-D object points on the image plane and the actual 2-D image points were typically in the range of $0.5-0.7 \%$ (with $1.6-2.0 \%$ maximum errors). The object (robot arm) distance from these cameras was about $3 \mathrm{~m}$, and the $0.6 \%$ average error on the image plane corresponds to 1 $\mathrm{cm}$ displacement error on the hypothetical plane $3 \mathrm{~m}$ in front of the camera. Two zoom settings were used for the overhead (front-view) camera, which was about $1 \mathrm{~m}$ away from the robot end effector. For the wide-angle view (fovy $=38^{\circ}$ ), the average error on the image plane was typically $0.7-0.9 \%(2.0-2.8 \%$ maximum error), and the 


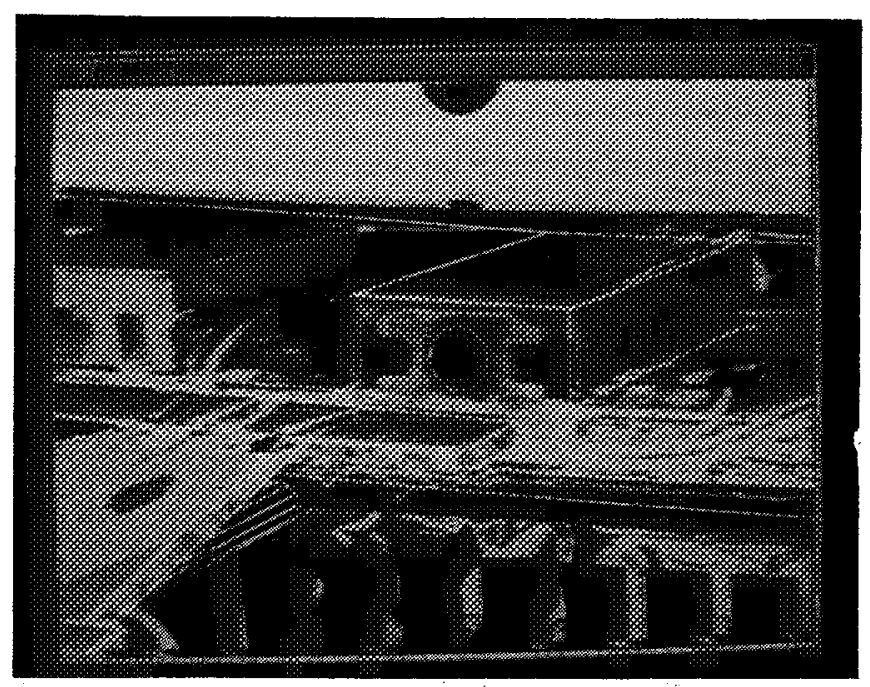

Fig. 6. Same configuration and TV camera view as in Fig. 5, but camera zoomed in.

$0.8 \%$ average error corresponds to $0.5 \mathrm{~cm}$ displacement error on the plane $1 \mathrm{~m}$ in front of the camera. For the zoom-in view (fovy $=8^{\circ}$ ), the average error on the image plane was typically $1.2-1.6 \%(3.2-$ $4.2 \%$ maximum error), and the $1.4 \%$ average error corresponds to $0.2 \mathrm{~cm}$ displacement error on the plane $1 \mathrm{~m}$ in front of the camera (See Fig. 6 for this setting).

The average object localization errors on the image planes were about $0.9-1.9 \%$ (3.0-7.0\% maximum errors). The $1.4 \%$ average error on the image planes corresponds to $0.2 \mathrm{~cm}$ displacement error with respect to the zoom-in overhead camera view, and to $2.5 \mathrm{~cm}$ displacement error with respect to the side-view or oblique-view cameras, in wide field-of-view settings.

\section{CONCLUSION}

The demonstration experiments have been performed successfully, showing the practical utility of high-fidelity predictive/preview display techniques, combined with compliance control, for the type of telerobotic servicing tasks in space that were shown in the May, 1993 demonstration. The same techniques also have a wide range of terrestrial application possibilities. Future work will include: 1) simulated tests on other space application tasks like Hubble Space Telescope Servicing, and 2) interactive model building and intermittent model matching updates using model-based image processing.

\section{ACKNOWLEDGMENT}

The contributions of H. Das, E. Paljug, and E. Barlow of JPL and S. Leake and E. Cheung of GSFC are gratefully acknowledged. Thanks also go to S. Ollendorf of GSFC for making available the GSFC staff and facilities, and for helping organizing the work.

\section{REFERENCES}

[1] A. K. Bejczy and W. S. Kim, "Predictive displays and shared compliance control for time-delayed telemanipulation," in Proc. IEEE Int. Workshop on Intelligent Robots and Systems (IROS '90), Tsuchiura, Japan, July 1990 , pp. 407-412.

[2] W. S. Kim and A. K. Bejczy, "Graphics displays for operator aid in telemanipulation," in Proc. IEEE Conf. Syst., Man, Cybern., Charlottesville, VA, Oct. 1991, pp. 1059-1067.

[3] W. S. Kim, B. Hannaford, and A. K. Bejczy, "Force-reflection and shared compliant control in operating telemanipulators with time delay," IEEE Trans. Robotics Automat., vol. 8, no. 2, pp. 176-185, 1992.

[4] W. S. Kim, "A reliable operator-interactive camera calibration and object localization method for predictive/preview displays in telerobotics," JPL Document, to appear in Dec. 1993.

[5] T. B. Sheridan, "Space teleoperation through time delay: Review and prognosis," IEEE Trans. Robotics Automat., vol. 9, pp. 592-606, Oct. 1993. 
